\title{
Optimal SINR Based Resource Allocation For Simultaneous Energy and Information Transfer
}

\author{
Ghada Saleh, C. Emre Koksal, and Ness B. Shroff \\ Department of ECE, Ohio state University \\ Email: \{salehg,koksal,shroff\} @ece.osu.edu
}

\begin{abstract}
In this paper, we study the problem of simultaneous information and power transfer in a wireless multiuser, single hop network. Specifically, we develop an optimal resource allocation algorithm that maximizes the long term average rate of the network while harvesting energy from both the received signal and the interference. We focus on receivers that split the received signal power into two streams; one is fed to an information decoding block and the other is fed to an energy harvester block. We use a weight perturbation Lyapunov approach to optimally schedule data admission and allocate the powers and the energy splitting factors. We show that the designed algorithm achieves $\mathcal{O}(1 / K)$ of the optimal and requires $\mathcal{O}(K)$ data queue and battery size.
\end{abstract}

\section{Keywords}

Energy Harvesting, Resource Allocation, Power Consumption.

\section{INTRODUCTION}

The last few years has witnessed a rapid proliferation of untethered devices, which coupled with the dramatic increases in powerful new applications has resulted in significant energy demands on wireless devices. Thus, fueling research that addresses the problem of prolonging the operation without being tethered to an outlet for recharging. Such a problem has led to the focus on developing devices that are able to simultaneously communicate information and harvest energy [1]. Two main approaches have been considered in designing energy harvesting networks. One approach is to use renewable sources such as wind, solar and geothermal. However, such resources depend on factors like weather, and location which are time-varying and not suitable for various scenarios (e.g., indoor communications). The other approach is to make use of the RF signals which are known to carry both information and energy. Such an approach is most useful in scenarios where, for instance, there exists an access point equipped with a powerful power source that can be used to recharge other devices within the same network. Energy-constrained communication systems such as sensor networks and RFIDs are other important applications of such an approach. In addition, indoor communication is known to suffer from high interference that can be made useful if harvested.

We consider a multi-user, single hop network, where simultaneous information and power transfer is considered. We focus on power splitting receivers and we assume that the energy harvester block can harvest the energy from the received signal and the interference. In such a scenario, a choice between half/full duplex energy transmission and half/full duplex data transmission needs to be made. Since interference is useful in harvesting the energy, we find that full-duplex energy transmission is more suitable because harvesting self-interference can introduce high energy gain. On the other hand, half duplex data transmission has been the dominant practical means for transmission. However, recent advances have been made in mitigating selfinterference and developing a real-time full-duplex physical layer and MAC layer, such as the work in [11], [12] and [13]. We solve the problem and provide an algorithm for the full-duplex setting, and then highlight changes required to modify the algorithm for half-duplex data transmission.

We take into account the decoding power required to successfully decode the received signal. We use the weight perturbation Lyapunov approach [14] to optimally schedule data admission and allocate the powers and the energy splitting factors with the objective of maximizing the sum of the utility of long term data rate of the users. We consider the case of infinite data queue and battery capacity and show that the designed algorithm achieves $\mathcal{O}(1 / K)$ of the optimal and requires $\mathcal{O}(K)$ data queue and battery size. Although the techniques used here are related to those developed in papers in [15] and others, our problem is fundamentally different because we focus on harvesting the energy from the received signal and make use of the interference from other signals and self-interference which make the problem more complex.

Varshney in [2] addressed the problem of simultaneous energy and information transfer and investigated the rateenergy trade-off over a single noisy link. This work was later extended in [3] to the case of frequency-selective channels with additive white Gaussian noise (AWGN). However the works in [2] and [3] considered a receiver that can simultaneously decode the received signal and harvest all its energy. Such a receiver is impractical because it is impossible to harvest all the received signal and simultaneously decode its content. The work in [4] considered a multiple-input multiple-output wireless broadcast network that consists of three nodes; an energy harvesting node, an information decoding node and a common receiver. The authors in [4] investigated the rate-energy trade-off of two practical receivers; (i) time switching receiver, in which, at any given time, either the information decoding node 
or the energy harvesting node is active, but not both; and (ii) power splitting receiver, in which the received signal is split into two streams; one is fed to an information decoder and the other is fed to an energy harvester. Orthogonal frequency division multiple access network was considered in [7], where a resource allocation algorithm has been designed to maximize the energy efficiency of data transmission. They considered power splitting receivers and assumed that the receiver can harvest the received signal as well as the interference. However they modeled the interference as AWGN which simplified the problem significantly because a power control algorithm, designed based on this model, does not take into consideration the effect of changing the power levels on interference.

On another front, the decoding power at the receiver can sometimes be large and cannot be ignored especially in energy-constrained networks. The authors in [8] highlight the importance of considering the decoding power when designing an energy efficient network. In [9], the authors study the decoding power of different error control coding schemes and show that, depending on the application, some codes can be unsuitable because of the high decoding power required. The authors in [10] found a lower bound on the decoding power and showed that large decoding power levels are required when operating close to capacity.

\section{PRoblem FORMULATION}

We consider a time-slotted multi-user, single-hop wireless network consisting of $N$ nodes. A user $i j$ is associated with a transmission from a node $i$ to a node $j$. Let $\mathcal{L}$ be the set of links $(i, j)$, where $L$ is the cardinality of the set $\mathcal{L}$. Each node is equipped with a receiver that can decode information and harvest received energy simultaneously. The harvested energy is stored in a battery. The received signal at the $j$ th receiver is split into two signals; $\rho_{j}$ fraction of the signal is fed to the energy harvester and $1-\rho_{j}$ fraction is fed

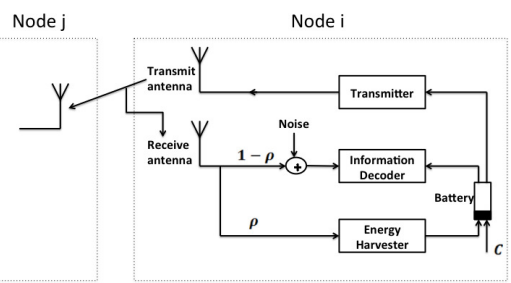
to the information decoder as shown in Fig. 1. Since we allow for full-duplex data transmission, a self-interference cancellation block is impeded in the information decoder.

At the beginning of time-slot $t$, the network determines the number of packets admitted to user $i j$, i.e, admitted to node $i$ and destined to node $j$. Let this number be $R_{i j}(t)$ and assume that $0 \leq R_{i j}(t) \leq R_{\max }$. Let $P_{i j}(t)$ be the power assigned to transmissions from node $i$ to $j$ in time slot $t$. We will assume that $P_{i i}(t)=0 \quad \forall i, t$ and $0 \leq$ $P_{i j}(t) \leq P_{\max } \forall i, j$. Let $\vec{P}=\left\{P_{i j},(i, j) \in \mathcal{L}\right\}$, and $\vec{\rho}=$ $\left\{\rho_{j}, j=1,2, \ldots, N\right\}$ be the vector of power assignments, and vector of splitting factors of the network, respectively.
The rate of transmission from node $i$ to node $j$ at time $t$ is denoted by $\mu_{i j}\left(\rho_{j}(t), \vec{P}(t)\right)$, where $\mu_{i j}$ is a function of the power assignment $P_{i j}(t)$, the interference from other transmissions $P_{m n}(t) \forall(m, n) \in \mathcal{L},(m, n) \neq(i, j)$ and the splitting factor $\rho_{j}(t) . \mu_{i j}(.,$.$) is assumed to be non-$ increasing in $\rho_{j}$, map $[0,1] \times \mathbb{R}_{>_{0}}^{L} \rightarrow \mathbb{R}_{\geq 0}$, and $\mu_{i j}(1, \vec{P})=0$.

Each node $i$ is equipped with a maximum of $N-1$ queues, one for each destination node $j$ such that $(i, j) \in \mathcal{L}$. The size of the queue at the $i$ th node with packets destined to the $j$ th node in the $t$ th time slot is denoted $Q_{i j}(t)$, where the queue dynamics are given as follows.

$$
Q_{i j}(t+1)=\left(Q_{i j}(t)-\mu_{i j}\left(\rho_{j}(t), \vec{P}(t)\right)\right)^{+}+R_{i j}(t)
$$

where $(.)^{+}=\max \{., 0\}$. The battery level at node $j$ in time-slot $t$ is denoted by $B_{j}(t)$, where the battery dynamics can be written as

$$
B_{j}(t+1)=B_{j}(t)-B_{j}^{(\text {out })}(t)+B_{j}^{(\text {in })}(t)
$$

where $B_{j}^{\text {(out) }}(t)$ is the power consumption at node $j$, and $B_{j}^{(\mathrm{in})}(t)$ is the harvested power at node $j$ plus some nonnegative energy drift $C_{j}$ that is fed to the battery at each time slot. Let $P_{D}\left(r_{i j}(t)\right)$ be the power required to decode a signal with rate $r_{i j}$. Hence,

$$
B_{j}^{\text {(out) }}(t)=\sum_{i} P_{D}\left(\mu_{i j}\left(\rho_{j}(t), \vec{P}(t)\right)\right)+\sum_{i} P_{j i}(t) .
$$

At this point we will assume that $P_{D}\left(\mu_{i j}\left(\rho_{j}(t), \vec{P}(t)\right)\right)=$ $P_{D_{u}} \mu_{i j}\left(\rho_{j}(t), \vec{P}(t)\right)$, where $P_{D_{u}}$ is the decoding power of a unit rate. Hence,

$$
B_{j}^{(\text {out })}(t)=\sum_{i} P_{D_{u}} \mu_{i j}\left(\rho_{j}(t), \vec{P}(t)\right)+\sum_{i} P_{j i}(t)
$$

Also $B_{j}^{(\text {in) }}(t)$ can be written as

$$
B_{j}^{(\mathrm{in})}(t)=C_{j}+\rho_{j}\left(\sum_{i} G_{i j} P_{i j}+\sum_{m, n \neq j} G_{m j} P_{m n}+\beta \sum_{i} P_{j i}\right)
$$

where $G_{i j}$ is the path loss of transmitting from node $i$ to node $j$, and we assume that $G_{i j}=\left(\frac{\lambda}{l_{i j}}\right)^{\gamma}$ where $l_{i j}$ is the distance between node $i$ and node $\stackrel{j}{j}, \lambda$ is the wavelength and $\gamma$ is the path loss exponent. The terms in (4), from left to right, are the non-negative energy drift, the received signal, the interference from other nodes and the self-interference. $\beta$ is the efficiency of harvesting the self-interference where $0 \leq \beta \leq 1$. Note that $B_{j}^{(\mathrm{in})}(t)$ and $B_{j}^{\text {(out) }}(t)$ can be upper bounded as follows.

$$
\begin{aligned}
B_{j}^{(\text {in })}(t) & \leq C+\left(L G_{\max }+(N-1) \beta\right) P_{\max }=: B_{\max }^{(\text {in })} \\
B_{j}^{\text {(out) }}(t) & \leq(N-1)\left(p_{D_{u}} r_{\max }+P_{\max }\right)=: B_{\max }^{\text {(out })}
\end{aligned}
$$

where $C=\max _{j}\left\{C_{j}\right\}, G_{\max }$ is the maximum path loss between any two nodes in the network, and

$r_{\max }=\max _{i, j}\left\{\mu_{i j}\left(\rho_{j}=0, P_{i j}=P_{\max }, P_{m n}=0 \forall(m, n) \neq(i, j)\right)\right\}$ 
The allocated powers need to not exceed the available battery level. In addition, we need the available plus the harvested energy to be at least equal to the total consumed power at any time. Thus, we have the following constraints.

$$
\begin{aligned}
& \sum_{i} P_{j i}(t) \leq B_{j}(t)+C_{j} \quad \forall j \\
& B_{j}^{\text {(out) }}(t) \leq B_{j}(t)+B_{j}^{(\text {in })}(t) \quad \forall j
\end{aligned}
$$

Note that for the half-duplex case, we have $B_{j}^{(\text {out })}(t)=$ $\sum_{i} P_{D_{u}} \mu_{i j}\left(\rho_{j}(t), \vec{P}(t)\right)$ or $B_{j}^{(\text {out })}(t)=\sum_{i} P_{j i}(t)$, so the allocated powers and splitting factors need to satisfy one of the above two constraints at any time slot not both.

The network aims to design data rate, power allocation, and splitting factors, $R_{i j}(t), P_{i j}(t), \rho_{j}(t) \forall i, j, t$, respectively, with the goal of maximizing the sum of the utility of the long term transmission rate of the users. Let $U\left(r_{i j}\right)$ be the utility of user $i j$ when transmitting with rate $r_{i j}$. $U($.$) is assumed to be strictly concave, non decreasing and$ continuously differentiable with bounded first derivative at zero, i.e., $U^{\prime}(0)<\infty$. The problem can be written as

$$
\begin{array}{ll}
\max _{\vec{R}(t), \vec{\rho}(t), \vec{P}(t)} & \sum_{i, j} U\left(\lim _{T \rightarrow \infty} \frac{1}{T} \sum_{t=0}^{T-1} R_{i j}(t)\right) \\
\text { subject to } \quad & \limsup _{T \rightarrow \infty} \frac{1}{T} \sum_{t=0}^{T-1} B_{j}(t)<\infty \quad \forall j \\
& \limsup _{T \rightarrow \infty} \frac{1}{T} \sum_{t=0}^{T-1} Q_{i j}(t)<\infty \quad \forall i, j
\end{array}
$$

(5) and (6)

where (8) and (9) guarantees the stability of the network. We let $\vec{R}^{*}$ be the optimal solution of the above problem and let $U^{*}=\sum_{i, j} U\left(R_{i j}^{*}\right)$.

\section{RESOURCE ALLOCATION ALGORITHM}

The resource algorithm uses the weight perturbation Lyapunov approach [14]. The idea is to carefully choose the perturbation value such that the energy availability constraint becomes redundant. However in our problem, we have two energy availability constraints. In addition, the constraint (6) introduce additional difficulty due to the large interaction between the power allocated on different links which makes the problem much more complex.

We first introduce the virtual queues $\tilde{Q}_{i j}(t)$ and $\tilde{B}_{j}(t)$ with the following dynamics.

$$
\begin{aligned}
\tilde{Q}_{i j}(t+1) & =\tilde{Q}_{i j}(t)-\left(1-1_{\alpha_{i}}(t)\right) \mu_{i j}(\vec{\alpha}(t)) \\
& -1_{\alpha_{i}}(t) \mu_{i j}\left(\rho_{j}(t), \vec{P}(t)\right)+R_{i j}(t) \\
\tilde{B}_{j}(t+1) & =\tilde{B}_{j}(t)-\left(1-1_{\alpha_{j}}(t)\right) \sum_{i} \alpha_{j i}(t)+C_{j} \\
& -1_{\alpha_{j}}(t) \rho_{j}(t) \sum_{i}\left(G_{i j} P_{i j}(t)+\sum_{n \neq j} G_{i j} P_{i n}(t)+\beta P_{j i}(t)\right) \\
& +1_{\alpha_{j}}(t)\left(\sum_{i} P_{j i}(t)+P_{D u} \sum_{i} \mu_{i j}\left(\rho_{j}(t), \vec{P}(t)\right)\right)
\end{aligned}
$$

where $\alpha_{i j}(t)$ is an auxiliary variable and we restrict $\alpha_{i j}(t)$ to satisfy $0 \leq \alpha_{i j}(t) \leq P_{\max }$, and

$$
1_{\alpha_{j}}(t)= \begin{cases}1 & \sum_{i} \alpha_{j i}(t)>0 \\ 0 & \sum_{i} \alpha_{j i}(t)=0\end{cases}
$$

Define the perturbed Lyapunov function as

$L(t):=\sum_{i, j} \tilde{Q}_{i j}^{2}(t)+\sum_{j}\left(\tilde{B}_{j}(t)-1_{\alpha_{j}}(t) b_{j}-\left(1-1_{\alpha_{j}}(t)\right) d_{j}\right)^{2}$

where $b_{j}$ and $d_{j}$ are the perturbation values and will be specified later. The overall solution requires solving the following sub-problems at each time t:

- Rate allocation (RA):

$$
\begin{aligned}
& \vec{R}(t)=\arg \max _{r_{i j}} \sum_{i, j}\left(K U\left(r_{i j}\right)-\tilde{Q}_{i j}(t) r_{i j}\right) \\
& 0 \leq r_{i j} \leq R_{\max } \quad \forall i, j
\end{aligned}
$$

- Energy Availability (EA):

$$
\begin{aligned}
& \vec{\alpha}(t)=\arg \max _{\vec{\alpha}}\left\{\sum_{i, j}\left(1-1_{\alpha_{i}}\right) \tilde{Q}_{i j}(t) \mu_{i j}(\vec{\alpha})\right. \\
& \left.\quad+\sum_{j}\left(1-1_{\alpha_{j}}\right)\left(\tilde{B}_{j}(t)-d_{j}\right)\left(\sum_{i} \alpha_{j i}-C_{j}\right)\right\} \\
& 0 \leq \alpha_{i j} \leq P_{\max } \forall i, j \\
& \sum_{i} \alpha_{j i} \leq B_{j}^{(\max )}(t)+C_{j} \quad \forall j
\end{aligned}
$$

- Power allocation and energy harvesting (PA-EH): if $1_{\alpha_{j}}=0$ set $P_{j i}(t)=0 \forall i$ and solve the following problem for $\vec{\rho}$ and $P_{m n}, \forall m \neq j, \forall n$. If $1_{\alpha_{j}}=1$ then solve the following problem for $\vec{\rho}$ and $\vec{P}$.

$$
\begin{aligned}
& {[\vec{\rho}(t), \vec{P}(t)] }=\underset{\vec{\rho}, \vec{P}}{\arg \max _{i, j}}\left\{\sum_{i j} \tilde{Q}_{i j}(t) \mu_{i j}\left(\rho_{j}, \vec{P}\right)\right. \\
&\left.+\sum_{j}\left(\tilde{B}_{j}(t)-b_{j}\right)\left(B_{j}^{(\text {out })}\left(\rho_{j}, \vec{P}\right)-B_{j}^{(\text {in })}\left(\rho_{j}, \vec{P}\right)\right)\right\} \\
& 0 \leq P_{i j} \leq P_{\max }, 0 \leq \rho_{j} \leq 1 \quad \forall i, j \\
& \sum_{i} P_{j i} \leq B_{j}(t)+C_{j} \quad \forall j \\
& B_{j}^{(\text {out })}(t) \leq B_{j}(t)+B_{j}^{(\text {in })}(t) \quad \forall j
\end{aligned}
$$

\section{PERformance EVAluation}

We now specify the values of $d_{j}$ and $b_{j}$ and evaluate the performance of the resource allocation algorithm. We first note that at any time $t, Q_{i j}(t)=\tilde{Q}_{i j}(t)$ and $B_{j}(t)=\tilde{B}_{j}(t)$. $b_{j}$ and $d_{j}$ are defined as follows.

$$
\begin{aligned}
d_{j} & =(N-1) P_{\max }-C_{j} \\
b_{j} & =B_{\max }^{\text {(out) }}-C_{j}+\frac{K U^{\prime}(0)+R_{\max }}{p_{D u}}
\end{aligned}
$$

Theorem 1: Under the values of $d_{j}$ and $b_{j}$ in (15) and (16), respectively, the resource allocation algorithm (12)(14) satisfies the following.

1) The data queue satisfy the following bound

$$
Q_{i j}(t) \leq K U^{\prime}(0)+R_{\max }=: Q_{\max } \quad \forall i, j, t
$$




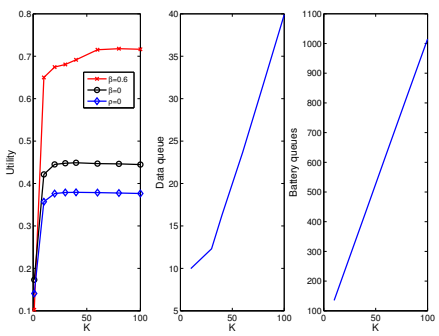

Fig. 2. Behavior of the network with different $K$ values
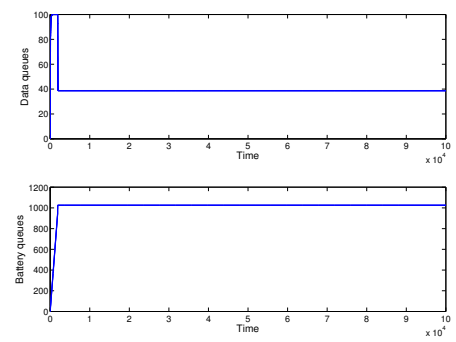

Fig. 3. Sample path processes of the data and energy queues

2) If $B_{j}(t)<d_{j}$ then $1_{\alpha_{j}}(t)=0$. Hence, the constraint (5) is always satisfied.

3) If $B_{j}(t)<B_{\max }^{\text {(out) }}-C_{j}$ then for any $P_{i j}(t)>0$ the associated splitting factor satisfies $\rho_{j}(t)=1$ and if $B_{j}(t)>b_{j}$ then $\rho_{j}(t)=0$. As a result, the constraint (6) is always satisfied.

4) We assume that $C_{j}=0$ when $B_{j}(t)>b_{j}$. The battery queue satisfy the following bound.

$$
B_{j}(t) \leq b_{j}+B_{\max }^{(\mathrm{in})}=: B_{\max } \forall j, t
$$

5) Let $\vec{r}$ be the time average of $\vec{R}(t)$ found using RA algorithm. We have the following bound.

$$
\liminf _{T \rightarrow \infty} \sum_{i, j} U(\vec{r}) \geq U^{*}-\frac{S}{2 K}
$$

where $S=L R_{\max }^{2}+L r_{\max }^{2}+N\left(\left(B_{\max }^{(\text {in })}\right)^{2}+\left(B_{\max }^{(\text {out })}\right)^{2}\right)$. The proof is omitted due to page limitations. The theorem implies that the achieved utility using the proposed algorithm is $\mathcal{O}(1 / K)$ of the optimal, and we only need data and battery queues with size $\mathcal{O}(K)$.

\section{NUMERICAL RESUltS}

We consider a network consisting of 3 nodes and 6 links. We assume that all nodes have the same energy drift $C_{1}=C_{2}=C_{3}=0.5$, The distance between the nodes is one meter. The frequency is $800 \mathrm{MHZ}$ and $\gamma=1.5$. Let $P_{\max }=2, P_{D u}=0.1, U(x)=\log (1+x)$, and $\mu_{i j}\left(\rho_{j}, \vec{P}\right)=W \frac{G_{i j} P_{i j}}{N_{o}+\sum_{m, n \neq j} G_{m j} P_{m n}}$ where $W=10$, and $N_{o}=1$ and $R_{\max }=3$. The simulation is run for $10^{5}$ time slots and $K=[1,10,20,30,40,60,80,100]$.

Fig. 2 compares the utility for the cases of $\beta=0.6$, $\beta=0$, and the case when no energy is harvested $(\rho=0)$ and shows the gain of harvesting self-interference. The data and battery queues increase linearly with $K$ and the utility increases with $K$ and converges to the optimal. Fig. 3 shows multiple sample path data and energy queue processes for $K=100$. The results coincides with bounds in Theorem 1 .

\section{CONCLUSION}

In this paper, we developed an optimal resource allocation algorithm for a network in which each user transfers information and energy simultaneously. Each receiver harvests a portion, chosen optimally, of the received signal and interference energy. The proposed algorithm achieves a utility arbitrarily close to $\mathcal{O}(1 / K)$ of the optimal.

\section{REFERENCES}

[1] L. Mateu, F. Moll et al., "Review of energy harvesting techniques and applications for microelectronics," in Proc. SPIE, vol. 5837, 2005, pp. 359-373.

[2] L. R. Varshney, "Transporting information and energy simultaneously," in Information Theory, 2008. ISIT 2008. IEEE International Symposium on. IEEE, 2008, pp. 1612-1616.

[3] P. Grover and A. Sahai, "Shannon meets tesla: wireless information and power transfer," in Information Theory Proceedings (ISIT), 2010 IEEE International Symposium on. IEEE, 2010, pp. 2363-2367.

[4] R. Zhang and C. K. Ho, "Mimo broadcasting for simultaneous wireless information and power transfer," in Global Telecommunications Conference (GLOBECOM 2011), 2011 IEEE. IEEE, 2011, pp. 1-5.

[5] X. Zhou, R. Zhang, and C. K. Ho, "Wireless information and power transfer: Architecture design and rate-energy tradeoff," arXiv preprint arXiv:1205.0618, 2012.

[6] L. Liu, R. Zhang, and K.-C. Chua, "Wireless information transfer with opportunistic energy harvesting," in Information Theory Proceedings (ISIT), 2012 IEEE International Symposium on. IEEE, 2012, pp. 950-954.

[7] D. W. K. Ng, E. S. Lo, and R. Schober, "Wireless information and power transfer: Energy efficiency optimization in ofdma systems," arXiv preprint arXiv:1303.4006, 2013.

[8] C. Xiong, G. Y. Li, Y. Liu, and S. Xu, "When and how should decoding power be considered for achieving high energy efficiency?" in Personal Indoor and Mobile Radio Communications (PIMRC), 2012 IEEE 23rd International Symposium on. IEEE, 2012, pp. $2427-2431$.

[9] S. L. Howard, C. Schlegel, and K. Iniewski, "Error control coding in low-power wireless sensor networks: When is ecc energy-efficient?" EURASIP Journal on Wireless Communications and Networking, vol. 2006, 2006.

[10] P. Grover, K. Woyach, and A. Sahai, "Towards a communicationtheoretic understanding of system-level power consumption," Selected Areas in Communications, IEEE Journal on, vol. 29, no. 8, pp. $1744-1755$, september 2011.

[11] J. I. Choi, M. Jain, K. Srinivasan, P. Levis, and S. Katti, "Achieving single channel, full duplex wireless communication," in Proceedings of the sixteenth апnиal international conference on Mobile computing and networking. ACM, 2010, pp. 1-12.

[12] N. Singh, D. Gunawardena, A. Proutiere, B. Radunovic, H. V. Balan, and P. Key, "Efficient and fair mac for wireless networks with self-interference cancellation," in Modeling and Optimization in Mobile, Ad Hoc and Wireless Networks (WiOpt), 2011 International Symposium on. IEEE, 2011, pp. 94-101.

[13] M. Jain, J. I. Choi, T. Kim, D. Bharadia, S. Seth, K. Srinivasan, P. Levis, S. Katti, and P. Sinha, "Practical, real-time, full duplex wireless," in Proceedings of the 17th annual international conference on Mobile computing and networking. ACM, 2011, pp. 301-312.

[14] L. Huang and M. J. Neely, "Utility optimal scheduling in energy harvesting networks," in Proceedings of the Twelfth ACM International Symposium on Mobile Ad Hoc Networking and Computing. ACM, 2011, p. 21.

[15] X. Lin, N. B. Shroff, and R. Srikant, "A tutorial on cross-layer optimization in wireless networks," Selected Areas in Communications, IEEE Journal on, vol. 24, no. 8, pp. 1452-1463, 2006. 